\title{
A propos des inondations catastrophiques de Nîmes
}

\author{
Jean-Claude Hémain \\ Laboratoire central \\ des Ponts-et-Chaussées
}

\author{
Christine Dourlens
}

Centre d'étude et de recherche

sur les pratiques de l'espace

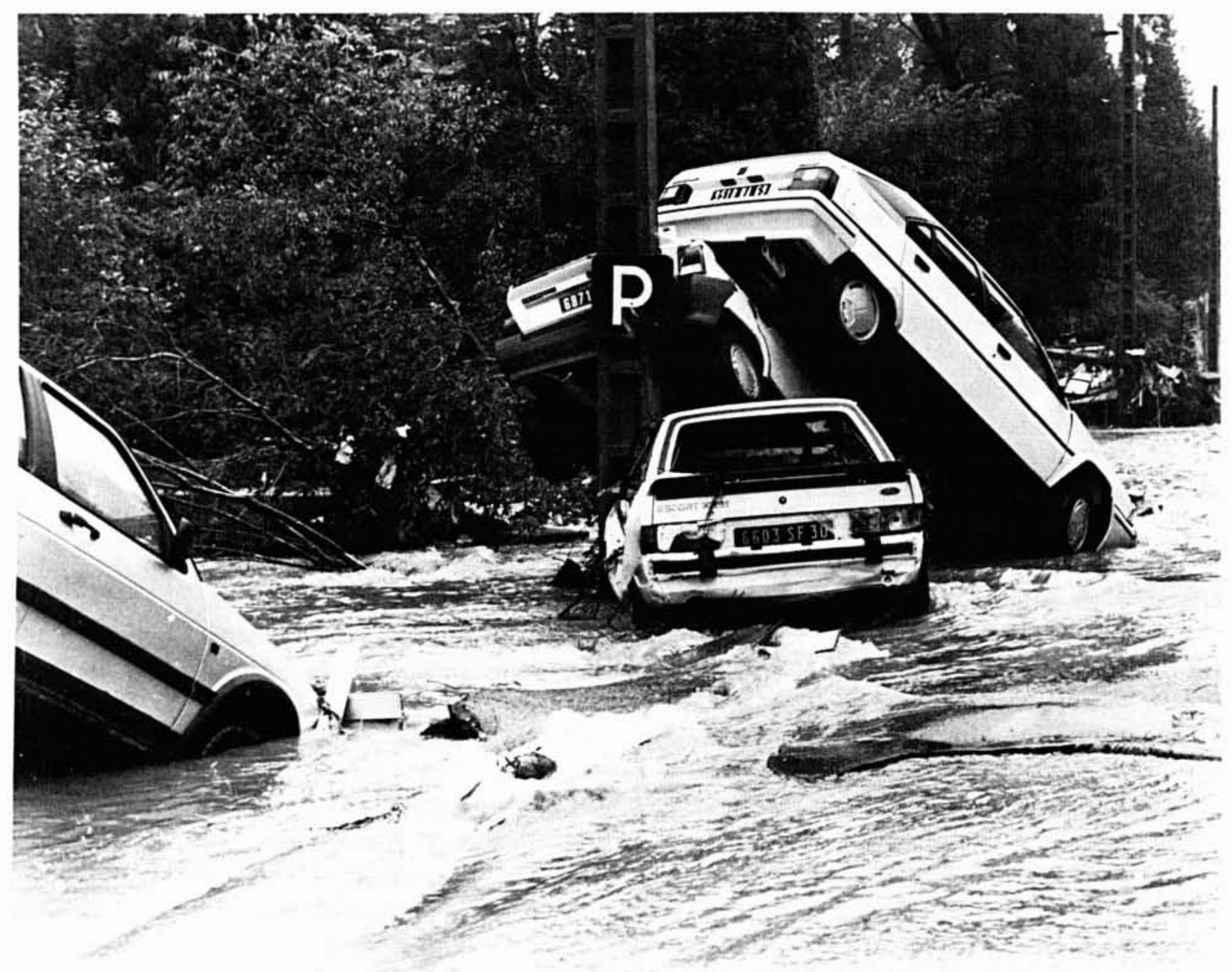

Le 3 octobre 1988, la ville de Nîmes a subi des précipitations exceptionnelles qui ont conduit à des dommages mémorables. La section Hydrologie urbaine de la Société hydrotechnique de France, au cours de la réunion qu'elle a tenue en novembre 1988, n'a pas manqué d'évoquer cet important événement. Il a paru intéressant de rassembler ici les exposés qui lui ont été consacrés, et qui concernent d'une part une première analyse des données pluviométriques disponibles quelques jours après la catastrophe, et d'autre part les réflexions qu'elle pouvait provoquer sur la gestion des risques majeurs. (photo Ph. Roussière, Midi Libre). 


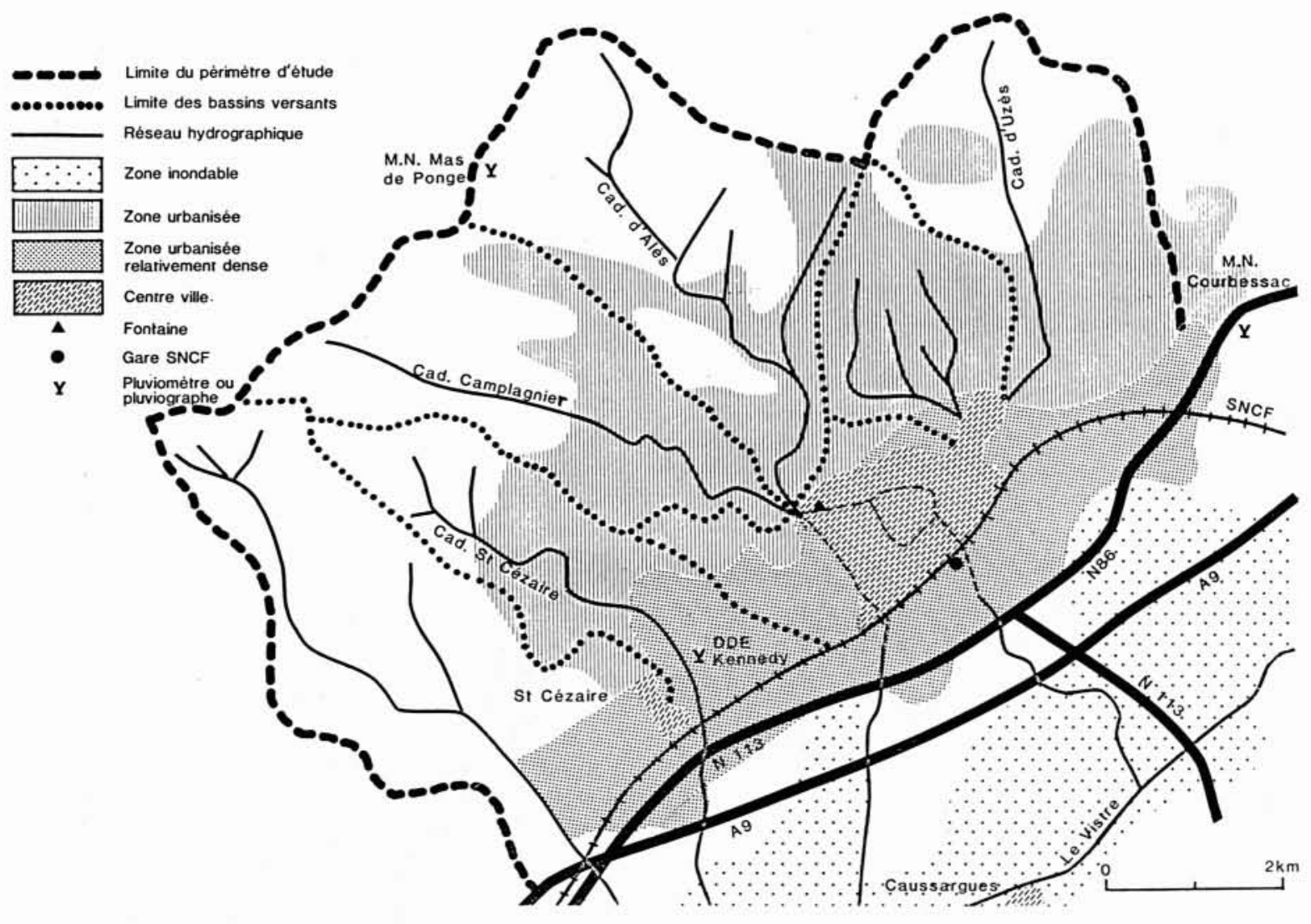

1. Contexte naturel et urbanisation.

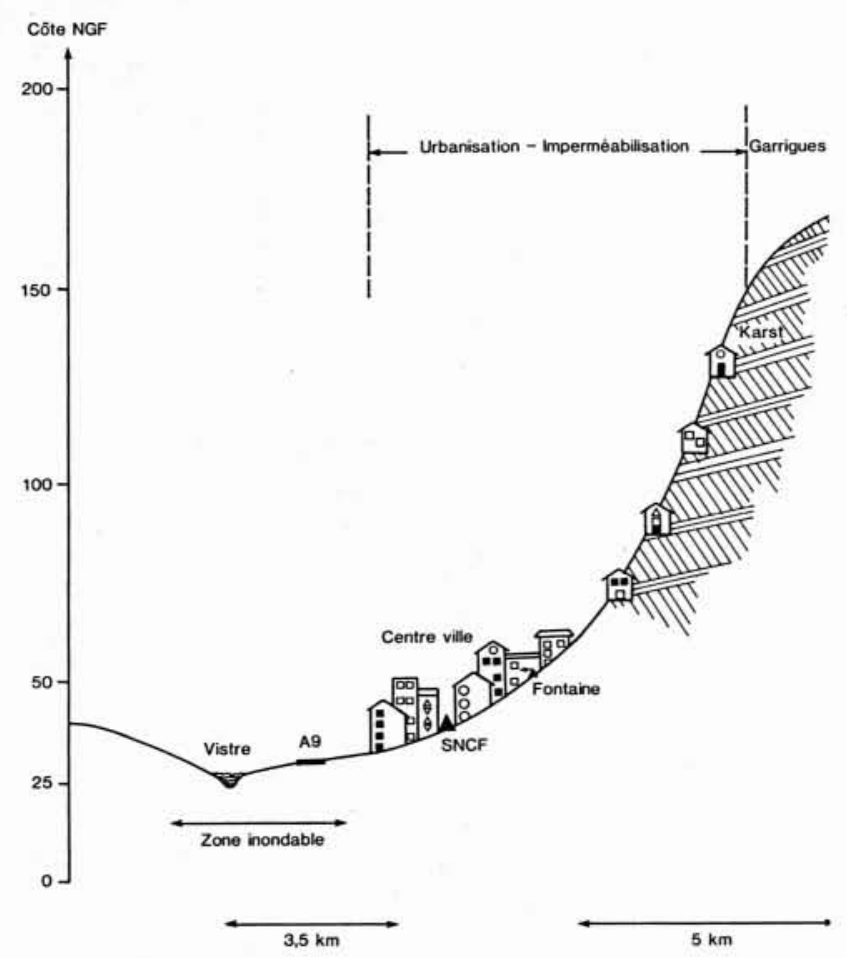

2. Coupe schématique. 


\title{
L'événement du 3 octobre 1988
}

\author{
par Jean-Claude Hémain
}

Je vais essayer de faire un résumé succinct de ce que l'on connaît à l'heure actuelle sur ce qui s'est passé à Nîmes le 3 octobre 1988, sur un plan technique, en termes d'information météorologique.

\section{Contexte géographique}

Pour situer le problème, voilà, très schématiquement, la description géographique de la ville telle qu'elle est schématisée sur le plan de la figure $l$ :

- le centre-ville :

- la zone urbanisée relativement dense ;

- les contours délimitant la zone complètement ou partiellement urbanisée ;

- les limites des bassins versants couvrant la ville et sa périphérie. On peut remarquer que ces limites sont relativement proches du centre-ville : il y a en gros 4 à $5 \mathrm{~km}$ entre l'entrée du centre-ville et les limites amont des bassins versants. Par contre, la dénivelée est importante : entre 100 et $150 \mathrm{~m}$.

Nous avons une structure en amphithéâtre, avec la ville qui s'est développée en bas, le long des grands axes de circulation qui constituent autant de barrages à l'écoulement :

- d'une part, la voie SNCF qui passe en centre-ville ou presque ;

- ensuite les Routes Nationales 86 et 113 qui sont devenues maintenant dans cette zone le périphérique sud de Nìmes ;

- enfin, l'autoroute A9.

\section{Le réseau hydrographique comprend:}

- les ruisseaux amont, les fameux Cadereaux, et notamment ceux d'Alès, de Complagnier et d'Uzès, qui ont été incriminés dans les inondations du Centre-Ville, puisqu'ils doivent le traverser en empruntant le réseau souterrain. Les conditions d'écoulement dans les lits amont des Cadereaux ont été profondément modifiées, autant dire le plus souvent détériorées, par le développement récent de l'urbanisation (voiries transversales, constructions dans les lits mineurs);

- complètement à l'aval, le Vistre, exutoire naturel de toute la zone : c'est un petit ruisseau de pente très faible et donc de capacité tout à fait ridicule par rapport au débit que peut lui apporter la ville en période de pluie importante ;

- autour du Vistre, par conséquent, une zone inondable. Voilà à peu près le cadre géographique.

D'un point de vue topographique, et encore de façon schématique (figure 2) :

- le Vistre se situe à peu près à $25-30 \mathrm{~m}$ d'altitude :

- les crêtes des bassins versants, sur les garrigues, fluctuent entre $150 \mathrm{~m}$ et $200 \mathrm{~m}$;

- l'entrée du Centre-ville, à peu près à hauteur de la fontaine, est à la cote $50-55 \mathrm{~m}$.

Vous imaginez donc les fortes pentes, d'une part, ainsi que l'urbanisation qui s'est développée, pour l'ęssentiel, sur ces pentes-là, la rupture de pente amont se situant à peu près à la limite des zones actuellement imperméabilisées.

Voilà donc réunies beaucoup de conditions pour connâ̂tre de grosses difficultés le jour où un événement conséquent survient.

\section{Principales données disponibles}

Abordons maintenant, pour aller vite, les informations pluviométriques dont nous disposons. Il s'agit essentiellement des relevés chronologiques réalisés en trois stations positionnées sur la figure 1 :

- la première est celle de Nîmes-Courbessac, station principale du réseau de la Météorologie nationale. Elle est située à l'Est de la Ville dans la zone la moins touchée en termes de hauteur pluviométrique et d'intensité. Les enregistrements ont été assurés correctement par le pluviographe jusqu'à 8 h $30-9$ h, après quoi, suite à une panne générale d'électricité, on ne dispose plus que de quelques relevés cumulés (comme, c'est malheureusement trop souvent le cas dans de telles situations, pour nous les plus intéressantes) ;

- la deuxième station dite "Kennedy " est gérée par la DDE et se situe dans la ZUP près de Saint-Cézaire. Des relevés horaires particulièrement intéressants $y$ ont été effectués ;

- la troisième station, celle du Mas de Ponge, est constituée d'un pluviomètre totalisateur relevé quotidiennement à $8 \mathrm{~h}$ par la Météorologie Nationale. Il est situé à l'extrémité amont des bassins versants des Cadereaux d'Alès et de Camplagnier. C'est le poste qui a mesuré les hauteurs précipitées les plus importantes. 
Tableau 1: LES MESURES DE PLUIE (mm)

\begin{tabular}{|c|c|c|c|}
\hline Station & $\begin{array}{c}\text { M.N. } \\
\text { Courbessac }\end{array}$ & $\begin{array}{c}\text { DDE } \\
\text { Kennedy }\end{array}$ & $\begin{array}{c}\text { M.N. } \\
\text { Mas de Ponge }\end{array}$ \\
\hline $\begin{array}{l}02 / 1022 \mathrm{~h} \\
\text { Le } 03 / 10 \mathrm{a}:\end{array}$ & 4,6 & 8,5 & Le $02 / 10$ à $8 \mathrm{~h}$ \\
\hline $4 \mathrm{~h}$ & 7 & 1,5 & \\
\hline $5 \mathrm{~h}$ & & 12,5 & \\
\hline $6 \mathrm{~h}$ & & 39 & \\
\hline $7 \mathrm{~h}$ & 35 & 47,5 & \\
\hline $8 \mathrm{~h}$ & & 46 & 200 \\
\hline $9 \mathrm{~h}$ & & 41,5 & \\
\hline $10 \mathrm{~h}$ & 160 & 37 & \\
\hline $11 \mathrm{~h}$ & & 42 & \\
\hline $11 \mathrm{~h} 30$ & & & \rfloor$>220^{\star}$ \\
\hline $12 \mathrm{~h}$ & & 43,5 & \\
\hline $12 \mathrm{~h} 30$ & 68 & & \\
\hline Total $03 / 10$ & 263 & 310,5 & $>420$ \\
\hline
\end{tabular}

Tableau 2 : HAUTEURS MAXIMALES DE PLUIE $(\mathrm{mm})$ Ev. du $03 / 10 / 88$

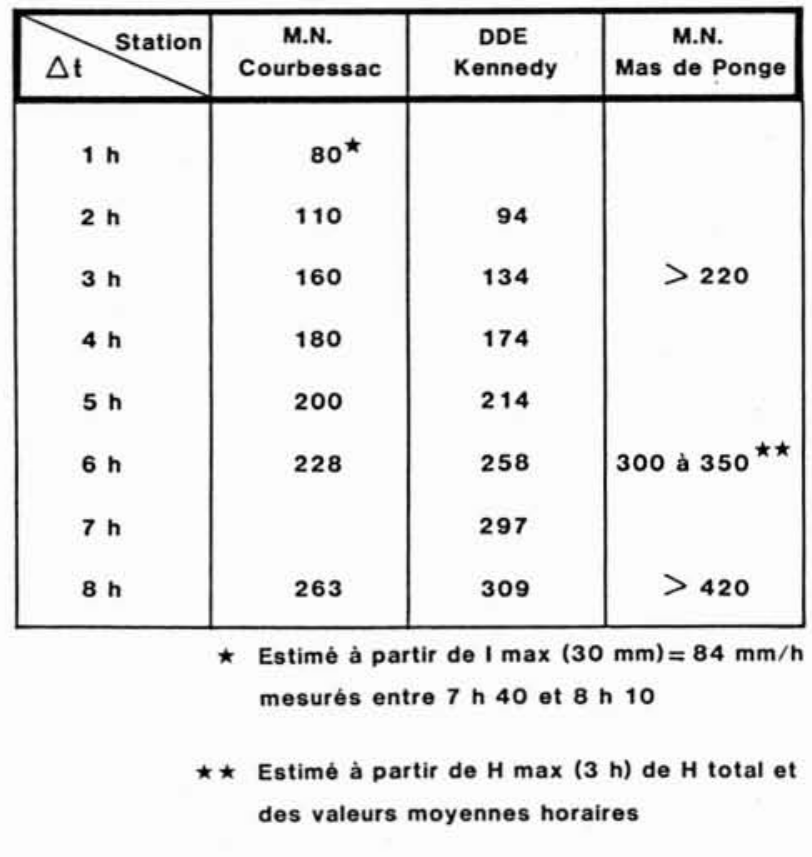

Les données disponibles sont rassemblées dans le tableau 1 .

On peut remarquer la régularité des précipitations horaires, entre $6 \mathrm{~h}$ et $12 \mathrm{~h}$.

Ces données brutes peuvent être exploitées en première analyse pour tenter d'estimer grossièrement les débits de pointes générés par cet événement pluvieux.

Pour ce faire, nous avons utilisé la formule rationnelle en évaluant les temps de concentration (après saturation des sols) à partir des caractéristiques physiques (surface, longueur, pente moyenne) des bassins versants concernés. Les valeurs obtenues varient de 25 à $45 \mathrm{mn}$.

L'incertitude liée à ces valeurs n'a finalement guère d'importance puisqu'on a enregistré des intensités très fortes pendant plusieurs heures avant d'observer les débits les plus élevés, à partir de $8 \mathrm{~h}$ ou $9 \mathrm{~h}$. La zone amont est alors largement saturée et la pluie s'est poursuivie avec des intensités comparables.

Les débits que je vais vous donner ont été établis en considérant qu'on récupère environ $80 \%$ du volume précipité sur la durée du temps de concentration. Le coefficient moyen d'imperméabilisation des sols est bien sûr nettement inférieur à ce chiffre. Mais là encore, on peut considérer qu'après plusieurs heures sous des intensités de 50 à $60 \mathrm{~mm} / \mathrm{h}$, les garrigues ont ruisselé comme les zones imperméables.

Premier chiffre donc : à l'entrée du Centre-Ville, sur les deux ovoïdes de capacité $7 \mathrm{~m}^{3} / \mathrm{s}$ et qui constituent l'exutoire des Cadereaux d'Alès et de Camplagnier, il est arrivé 250 à $300 \mathrm{~m}^{3} / \mathrm{s}$. Certains ont dit $400 \mathrm{~m}^{3} / \mathrm{s}$. Ces différences n'ont pas beaucoup d'importance, nous avons un ordre de grandeur.

A l'est, pour le Cadereau d'Uzès, on arrive à $100 \mathrm{~m}^{3} / \mathrm{s}$ environ, avec des capacités d'évacuation de $25 \mathrm{~m}^{3} / \mathrm{s}$, peut être $30 \mathrm{~m}^{3} / \mathrm{s}$.

Pour les bassins périphériques intermédiaires, tout à fait comparable à celui du Cadereau d'Uzès, les chiffres sont du même ordre.

Enfin, sur la ville elle-même, rapidement devenue un bassin de rétention, la pluie correspond à un débit de 100 à $120 \mathrm{~m}^{3} / \mathrm{s}$.

Au total, le débit qui a dû traverser le Centre-Ville, estimé à 700 ou $800 \mathrm{~m}^{3} / \mathrm{s}$ par des personnalités locales, serait selon nos hypothèses de 550 à $650 \mathrm{~m}^{3} / \mathrm{s}$. Face à ces chiffres, il faut rappeler qu'il n'y a pas de canal à ciel ouvert dans le réseau principal de Nìmes. Tout est busé et enterré.

Le problème de la Fontaine qui a été évoqué comme pouvant être l'une des causes des inondations se situe à plusieurs ordres de grandeur en dessous : son débit maximal, en crue, est de 20 à $30 \mathrm{~m}^{3} / \mathrm{s}$; le canal aval, à ciel ouvert sur quelques centaines de mètres, à une capacité de $50 \mathrm{~m}^{3} / \mathrm{s}$. Ce qui s'est probablement passé, c'est que les flots en provenance des Cadereaux, et qui n'ont pu s'écouler dans le réseau, se sont dirigés vers la fontaine et ont submergé le canal.

Voilà les ordres de grandeur. Il est clair qu'on n'est plus du tout, là, dans le domaine de la comparaison des débits ruisselés avec les capacités d'évacuation du réseau. Reste maintenant à savoir si ce genre de situation est aussi exceptionnel qu'on a bien voulu le dire. 
Tableau 3 : ESTIMATION DES PERIODES DE RETOUR (années)

DES HAUTEURS MAXIMALES PONCTUELLES

(station de référence : Nimes Courbessac)

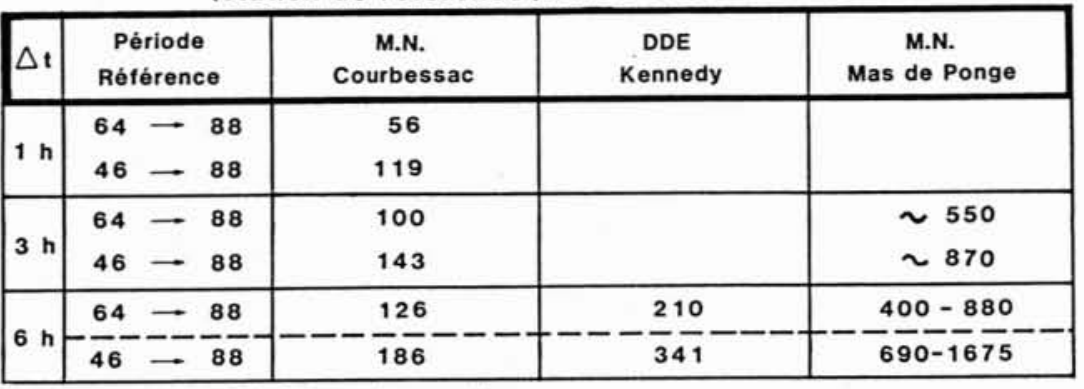

Valeurs > seuils, loi Gamma incomplète

\begin{tabular}{|c|c|c|c|}
\hline M.N. & 350 & 900 & $>5000$ \\
M. MERLIN & 650 & & $>5000$ \\
L H M 1 & 500 & 1700 & $550-1200$ \\
L H M 2 & 180 & 300 & $700-2000$ \\
L H M 3 & 200 & 350 & \\
\hline
\end{tabular}

$H$ max $(6 h)$ annuels, série $46 \rightarrow 88$

M.N. , M. MERLIN , L H M 1 : Gumbel

L H M 2 : Jenkinson

L H M 3: Log Normale

3. Premières estimations d'une "période de retour " de l'événement :

Je vais vous présenter maintenant non pas le fruit de réflexions personnelles mais le résultat d'un travail effectué principalement par Michel DESBORDES, du Laboratoire d'hydrologie mathématique de Montpellier (LHM), à qui, dans le cadre d'une commission "Hydraulique " mise en place par la Mairie de Nîmes, on a demandé d'estimer la période de retour de cet événement du 3 octobre 1988.

Les données de référence par lesquelles on peut essayer de calculer les périodes de retour sont rassemblées dans le tableau 2.

Elles sont issues des relevés mentionnés précédemment. Les hauteurs maximales sont de l'ordre de $100 \mathrm{~mm}$ sur $2 \mathrm{~h}$, ce qui n'est pas encore trop grave. Ce qui par contre est plus rare, c'est qu'elles croissent presque linéairement avec le pas de temps jusqu'à plus de $200 \mathrm{~mm}$ en $5 \mathrm{~h}$ ou $260 \mathrm{~mm}$ en $6 \mathrm{~h}$.

Je passe sur les détails du mode d'obtention de ces chiffres. Les uns peuvent être considérés comme fiables, quand on cumule des valeurs horaires, comme sur le poste de la DDE par exemple, d'autres qui sont des estimations, au Mas de Ponge notamment. Mais en gros les fourchettes sont relativement étroites; les incertitudes sont assez faibles, si on continue à raisonner en ordre de grandeur.

\subsection{Analyse ponctuelle}

Voilà une première méthode d'analyse : elle consiste à utiliser de façon classique les séries chronologiques pour ajuster des lois de probabilité sur divers paramètres caractéristiques des précipitations (courbes IntensitéDurée-Fréquence). On utilise ensuite ces lois pour affecter une probabilité, et donc une période de retour, à la valeur observée d'un paramètre.

Dans notre cas, la station de référence est celle de NîmesCourbessac. Elle est équipée d'un pluviographe depuis 1964 et, même s'il y a eu un changement d'appareil depuis cette date (comme dans la plupart des stations de la Météorologie nationale), on a une série relativement homogène de 25 années d'observations.

Auparavant, et depuis 1946, étaient effectués des relevés pluviométriques toutes les $12 \mathrm{~h}$. Bien que d'une précision moindre, ces informations sont exploitables si l'on s'intéresse à des pas de temps de plusieurs heures, dans la mesure où étaient notés les instants de début et de fin de pluie.

Les périodes de retour calculées pour les 3 stations étudiées et sur les séries de référence 1964-1988 et 19461988 sont données dans le tableau 3.

Ces résultats ont été obtenus en ajustant sur les séries observées des hauteurs d'eau maximales précipitées (pendant un intervalle de temps) supérieures aux seuils retenus par la Météorologie nationale, non pas une loi de GUMBEL "standard " mais la loi de probabilité la plus adaptée au sens des méthodes classiques de l'analyse statistique.

Vous voyez que ces périodes de retour oscillent :

- pour Courbessac, entre 100 ans et 200 ans pour les durées de $2 \mathrm{~h}$ à $6 \mathrm{~h}$; 
Tableau 4 : PLUIES REMARQUABLES OBSERVEES DEPUIS 1940

EN LANGUEDOC-ROUSSILLON

Hauteurs maximales $(\mathrm{mm})$ sur $\Delta t$

\begin{tabular}{|c|c|c|c|c|c|}
\hline$\Delta \mathrm{t}$ & $\mathrm{H}$ & Date & Lieu & Dépt. & Source \\
\hline $1 \mathrm{~h}$ & 94 & 23.09 .76 & St Gély du $\mathrm{F}$. & 34 & LHM \\
$1 \mathrm{~h}$ & 103 & & Perpignan & 66 & Rem. \\
$2 \mathrm{~h}$ & 140 & 30.09 .58 & Alès & 30 & CNABRL \\
$2 \mathrm{~h}$ & 190 & 12.10 .71 & St Gély du F. & 34 & LHM \\
$3 \mathrm{~h}$ & 312 & 13.10 .81 & Salanque & 66 & CNABRL \\
$4 \mathrm{~h}$ & 150 & 07.10 .79 & Montpellier & 34 & LHM \\
$4 \mathrm{~h}$ & 220 & 27.10 .76 & Vergeze & 30 & CNABRL \\
$4 \mathrm{~h}$ & 230 & 04.10 .58 & Quissac & 30 & CNABRL \\
$4 \mathrm{~h}$ & 240 & 16.10 .40 & Verney les B. & 66 & CNABRL \\
$4 \mathrm{~h}$ & 302 & 23.10 .76 & Les Matelles & 34 & LHM \\
$4 \mathrm{~h}$ & 356 & 13.10 .86 & Le Boulou & 66 & CNABRL \\
$5 \mathrm{~h}$ & 250 & 05.12 .87 & Aigues Vives & 34 & CNABRL \\
\hline $5 \mathrm{~h}$ & 250 & 03.10 .88 & Nimes & 30 & M.N. \\
$8 \mathrm{~h} / \mathrm{h}$ & 420 & 03.10 .88 & Nimes-Mas de Ponge & 30 & M.N. \\
\hline
\end{tabular}

Données très partielles ( 3 départements ,

- pour Kennedy, on monte un peu au-dessus de ces valeurs,

- pour Mas de Ponge, les chiffres sont plus impressionnants; mais il s'agit d'estimations tout de même plus douteuses. De plus, on se situe assez loin, et pas à la même altitude que la station de référence.

Une indication tout de même pour expliquer, sinon justifier, les chiffres qu'on a entendus jusque-là : la deuxième partie du tableau 3 montre les résultats qui ont été obtenus, uniquement sur des durées de $6 \mathrm{~h}$ et uniquement sur la série 1946-1988 (même si elle n'est pas tout à fait homogène). Les trois premières lignes de résultats correspondent à la même loi de valeurs extrêmes, à savoir la loi de Gumbel traditionnellement ajustée sur les hauteurs maximales annuelles. C'est une loi de probabilité à deux paramètres uniquement, en l'occurrence tout à fait inadaptée compte tenu de la forte disymétrie des échantillons.

Le premier chiffre est celui donné par la Météorologie nationale.

Le second est celui du cabinet Merlin, conseil de la Ville, qui a exploité les mêmes données.

Le troisième est celui qui a été obtenu par le LHM, toujours à partir des mêmes données.

Les seules différences sont les formules de fréquence empirique utilisées pour affecter une fréquence de non dépassement à une valeur observée. On peut déjà voir que la façon de travailler sur les mêmes données, même en ajustant une loi identique, peut aboutir à des contrastes notables.
Les deux chiffres figurant au-dessous ont été obtenus en utilisant d'autres lois qui diffèrent légèrement de la loi Gamma incomplète, mais qui, elles aussi, sont des lois à 3 paramètres qui peuvent s'ajuster sur des séries extrêmement dissymétriques. Les résultats sont légèrement différents de ceux mentionnés dans la première partie du tableau, mais découlent d'ajustements moins criticables à priori que celui de la traditionnelle loi de GuMBEL.

En conclusion, on peut déduire de ces analyses statistiques classiques, à partir d'une station de référence avec 25 ans de mesures homogènes, et sur les durées de pluie qui nous intéressent (entre $2 \mathrm{~h}$ et $6 \mathrm{~h}$ ) que l'on peut retenir des périodes de retour se situant entre 150 ans et 250 ans.

\subsection{Analyse régionale}

Une deuxième analyse a été faite par une approche différente qui a consisté à considérer les événements importants observés dans toute la région.

Les valeurs du tableau 4 sont au moins équivalentes sinon supérieures à celles qui ont été enregistrées sur Nîmes le 3 octobre et sur des durées de $1 \mathrm{~h}$ à $5 \mathrm{~h}$. Il s'agit de valeurs mesurées uniquement sur les parties basses de la Région Languedoc-Roussillon, dans les PyrénéesOrientales, le Gard et l'Hérault, depuis 1940 (altitude $<200 \mathrm{~m}$ ).

Trois sources d'informations seulement ont été compulsées. Les données rassemblées sont :

- celles disponibles à la Compagnie du Bas Rhône (CNABRL) ; 
- celles disponibles à l'Université de Montpellier (LHM) ;

- et quelques chiffres cités par RÉmÉnIÉRAs dans son ouvrage "L'hydrologie de l'ingénieur".

A la vue de ces valeurs, on constate que depuis 49 ans on a observé 13 événements d'importance au moins équivalente à celle de la pluie du 3 octobre 1988 à Nîmes :

- sans avoir compulsé toutes les données disponibles;

- en notant que, si l'on n'avait pas pris en compte l'année 1940, l'événement le moins récent serait celui de 1958 ; on aurait alors 12 événements sur une période de 21 ans.

En n'étant pas pessimiste, on a donc 13 événements de cette nature ou pire sur 49 ans, soit une période de retour, sur la région, d'au plus 4 ans.

On exploite alors cette information dans une simulation qui consiste à travailler sur les hypothèses suivantes :

- la surface concernée, délimitée comme on l'a vu, est à peu près de $8000 \mathrm{~km}^{2}$ (représentée par un rectangle $40 \mathrm{~km} \times 200 \mathrm{~km})$;

- on suppose qu'un orage de cette ampleur touche des surfaces circulaires de 300 à $500 \mathrm{~km}^{2}$;

- on suppose encore que ces orages peuvent se produire n'importe où, c'est-à-dire que leur loi de répartition géographique est uniforme, ce qui est loin d'être le cas car il y a des zones privilégiées pour ces orages importants. Ici encore, on tend à surestimer la valeur finale de la période de retour.

On va procéder à des simulations pour deux bassins versants fictifs, de $50 \mathrm{~km}^{2}$ et $80 \mathrm{~km}^{2}$ (sur Nîmes, la zone concernée était de $50 \mathrm{~km}^{2}$, sur d'autres villes, les surfaces peuvent être un peu plus grandes). En les situant chacun dans deux positions différentes, et en tirant au hasard 1000 situations pluviométriques (position de l'épicentre et surface touchée), on va compter le nombre de fois où les bassins sont touchés par la pluie sur plus de $80 \%$ de leur surface.

Ainsi de la période de retour d'un événement important en un point quelconque de la région, on pourra déduire la période de retour d'un événement de même nature touchant à + de $80 \%$ une surface donnée de $50 \mathrm{~km}^{2}$ ou $80 \mathrm{~km}^{2}$.

On arrive alors à des chiffres qui sont de l'ordre de 100 ans à 180 ans, sachant qu'on a maximisé ces valeurs notamment en adoptant une loi uniforme de répartition géographique et en considérant qu'en 49 ans il n'y a que 13 événements de cette ampleur sur la région.

\subsection{Analyse historique}

Troisième approche, aussi intéressante sinon plus, puisque ce qui nous préoccupe, c'est moins la pluie que ses conséquences et les dégâts qu'elle engendre: elle consiste à compulser les documents historiques.

Si l'on se réfère au seul ouvrage "Nîmes sans visa", de C. LIGER, c'est au moins la cinquième fois depuis 1399 , soit en 6 siècles environ, que les observateurs parlent de catas- trophe majeure ou d'apocalypse à propos des pluies sur Nîmes.

Cette voie mériterait d'être approfondie en consultant les archives de la ville (l'académie de Nîmes s'y emploie).

Même s'il faut rester prudent face à ce genre de discours ou d'écrit, il n'empêche que les informations se recoupent.

Par ailleurs, il faut savoir que d'autres événements cités dans l'ouvrage mentionné ci-dessus et que nous n'avons pas retenus, pourraient avoir aujourd'hui des conséquences catastrophiques compte tenu de l'évolution de la Ville qui a approximativement doublé depuis 20 ans, en population et en surface imperméabilisée. On pourrait ainsi facilement doubler le nombre des événements comparables à celui du 3 octobre 1988 et observés au cours des six derniers siècles.

Ce genre d'analyse est encore à approfondir, mais elle devrait aboutir à des périodes de retour de 100 ans au maximum.

\subsection{Bilan}

On retiendra que les études réalisées par le Laboratoire d'hydrologie mathématique visant à affecter une période de retour à l'événement du 3 octobre 1988 ont conduit aux valeurs suivantes:

- analyse de la série ponctuelle de Nîmes-Courbessac, série 1964-1988, pour un intervalle de temps de 2 à $6 \mathrm{~h}$ : $\mathrm{T}=150$ à 250 ans ;

- analyse des événements comparables observés dans la région et simulation par tirage au hasard: $\mathrm{T}=100$ à 180 ans ;

- analyse des écrits sur les "catastrophes historiques" : $\mathrm{T}=60$ à 120 ans.

Finalement, les ordres de grandeur sont tout à fait comparables : si on se réfère aux lois de probabilité ajustées sur la variable "hauteur maximale précipitée en $6 \mathrm{~h}$ " à Nîmes-Courbessac, la valeur de période de retour $\mathrm{T}=250$ ans n'excède que de $13 \%$ la valeur correspondante de période de retour $\mathrm{T}=150$ ans.

En conclusion, on peut affirmer que la période de retour de l'événement du 3 octobre 1988 est beaucoup plus proche de 100 ans que de 1000 ans.

\section{Conclusions}

Je viens de vous présenter des chiffres, des ordres de grandeur, mais que faut-il en retenir ?

Il est clair que la situation de Nìmes est particulière, on a cumulé à peu près tous les inconvénients, mais elle n'est pas unique. En tout état de cause, les discours du type "plus jamais ça " ou " on est tranquille pour 1000 ans ", tels qu'on a pu entendre sur TF1 le lendemain, méritent d'être révisés. La présentation suivante portera peut-être plus sur les enseignements à en tirer et sur l'analyse qui en a été faite, mais au plan technique, il faut retenir au moins ceci : 
1. - Jusqu'ici, on a toujours considéré que les ouvrages d'assainissement pluvial étaient des équipements mineurs pour l'infrastructure et la structuration de la Ville. Il est bien évident que tant qu'on continuera à raisonner ainsi on fera de grosses bêtises. C'est la première certitude.

2.- La deuxième, c'est que l'approche réglementaire, le niveau de protection "normalisé ", en gros la fréquence décennale et l'utilisation d'une technique " parisienne » qui résulte de l'histoire, constituent dans le midi de la France un danger majeur. On a des chiffres pour montrer que les situations locales ne correspondent pas. Il convient de s'aligner sur les connaissances contemporaines.

3. - Quant à la conséquence peut-être la plus importante, je dirai qu'il me paraît utile de considérer, comme le proposent certains Anglo-Saxons, deux niveaux dans l'assainissement :

- ce qui est du domaine de l'assainissement banal, qui constitue ce qu'on peut appeler le système mineur, et qui concerne l'équipement des zones nouvelles, des lotissements, des zones à faibles risques, où l'on peut utiliser des techniques classiques tout en réfléchissant tout de même aux données de pluviométrie locale;
- ce qui relève de l'assainissement exceptionnel, de l'exposition au risque d'inondation par ruissellement sur la ville. C'est un tout autre domaine. Ce n'est plus, pour moi de l'assainissement mais de l'aménagement de l'espace, de la structuration de la ville où on ne peut plus raisonner sans prendre en compte les contraintes d'évacuation des eaux pluviales (excédentaires par rapport aux capacités des systèmes d'assainissement).

C'est là sans doute l'information majeure sur le plan technique. Elle n'est pas nouvelle. Plusieurs personnes tiennent ce discours depuis longtemps et ici-même, à la dernière journée d'échange " Hydrologie urbaine SHF " de décembre 1987. Dommage qu'il aient été si peu écoutés jusqu'ici, mais on ne va pas refaire en quelques jours ce qui a été construit depuis plus d'un siècle.

Il faut repenser complètement la façon dont on restructure la Ville en imaginant ce qui peut se passer au moment d'événements pluvieux exceptionnels : par où passera, et fera-t-on passer, l'eau préférentiellement? quelle zone inondera-t-on, en premier lieu et le plus souvent ? etc...

C'est du domaine du choix politique, et plus tellement de notre ressort.

\title{
La gestion des risques majeurs
}

\author{
par Christine Dourlens
}

Je voudrais tout d'abord donner quelques précisions sur ce qui me conduit, en tant que sociologue, à m'intéresser à la gestion des risques urbains et à présenter aujourd'hui quelques réflexions à propos de la catastrophe de Nîmes du 3 octobre dernier.

Effectivement, rien d'évident, à priori, dans la mesure où la gestion des risques urbains liés à l'eau est essentiellement une affaire de spécialistes, une affaire d'ingénieurs. La protection des citadins, vis-à-vis de ce genre de danger, est en effet, depuis au moins le début du siècle, considérée comme un droit, droit garanti par les pouvoirs publics qui sont censés mettre en œuvre l'ensemble des compétences et des connaissances disponibles pour garantir la protection des citoyens.

L'objectif de l'action publique étant ainsi défini, sa mise en œuvre n'apparaît que comme une question technique. Il s'agit en fait de trouver les meilleurs moyens pour aboutir à une fin dont la définition semble s'imposer d'ellemême.
Il n'est pas étonnant alors, que dans ce contexte les sociologues se soient relativement peu investis dans ce domaine des risques urbains et qu'on ne les ait d'ailleurs que rarement associés, sinon pour leur demander d'analyser les comportements sociaux des populations face aux risques. Effectivement, l'étude de la perception sociale du risque est le domaine d'investigation réservé des sociologues. Et je dirai d'ailleurs que cette mise à contribution des sciences humaines pour analyser la subjectivité sociale face au risque tend à conforter une opposition entre ce qui serait de l'ordre du traitement scientifique et ce qui serait de l'ordre de l'irrationnel, de l'appréhension subjective de problèmes objectifs.

De façon un peu paradoxale, c'est cet état de fait qui laisse, en principe, peu de place aux questions d'ordre sociologique, si l'on excepte celles que pose la technique, qui a été l'aiguillon de notre curiosité. Et cela dans la mesure où le partage, dans une société, entre ce qui est relatif au politique, au technique et au social n'est pas une 
donnée à prendre comme telle. Au contraire ce partage est construit et s'élabore au cours des temps.

Alors, dans ce cas-là, pourquoi ne pas oser franchir les frontières disciplinaires qui reflètent un découpage social, somme toute, tout à fait contingent ? Pourquoi la sociologie n'aurait-elle pas à s'intéresser à des questions techniques? Et cela, pas seulement de l'extérieur, en étudiant par exemple les perceptions sociales de la technique, ou les transformations sociales induites par la technique. Mais aussi de l'intérieur en se demandant quelle rationalité est à l'œuvre dans l'appréhension purement technicienne d'un certain nombre de problèmes. Pourquoi la société confiet-elle à la science et à la technique le soin de régler des questions qui ne sont pas forcément seulement techniques? Quelle est en fait la signification d'un partage de rôles qui semble tellement évident qu'on le considère comme allant de soi, alors qu'il est produit historiquement?

Ceci étant posé, si la catastrophe de Nîmes m’intéresse c'est que justement en tant qu'événement de grande ampleur, parce qu'elle actualise un risque de probabilité relativement faible, elle agit un peu comme un événement perturbateur dans ce partage de rôles bien réglé entre technique, politique et social dans la gestion des risques urbains.

Plus précisément, elle met à jour le fait que l'adoption des mesures de protection, la définition des politiques de sécurité ne sont pas seulement techniques mais sont aussi l'expression de choix politiques qui n'ont pas d'autres références que celles que la société, elle-même, veut bien leur donner. La catastrophe de Nîmes révèle aussi le fait que le caractère politique de ces choix est, en temps ordinaire, relativement occulté.

Dans un premier temps, j'illustrerai le caractère politique des choix en matière de sécurité, et surtout le processus de dénégation du caractère politique de ces choix, en prenant l'exemple de l'adoption des dispositifs d'assainissement pluvial.

Adopter un dispositif d'assainissement pluvial, c'est fixer un seuil, un seuil de sécurité, se protéger contre les effets des pluies de période de retour 10,20,30,50 ans. Et c'est aussi choisir, finalement, entre un niveau de protection à l'égard d'un risque et l'effort à consentir, le prix à payer pour obtenir ce niveau de protection. Ce prix, d'ailleurs, n'est pas que monétaire : on choisit entre tel gain de sécurité et telle réalisation d'équipement sportif, culturel, scolaire que le coût de la protection empêchera de réaliser ; mais on choisit aussi entre ce gain de sécurité et des choses qui ne sont pas du tout monétarisables comme les coûts écologiques, les coûts paysagers, les coûts sociaux. Ceci implique finalement que la sécurité se gagne toujours sur quelque chose, et que, contrairement à l'adage, elle a un prix. Il y a toujours des arbitrages qui se font entre tel gain de sécurité, dans tel domaine, dans tel secteur et de tel point de vue, et tel autre objectif concurrent. Ceci implique aussi que tel niveau de protection, garanti à un moment donné, est aussi toujours un choix de non-protection.

Il est alors évident que l'arbitrage entre ces différents enjeux concurrents ne peut se faire au regard d'un quelconque optimum technique. Je dirais même que le choix d'un seuil de protection est en fait un choix entre des éléments de nature incomparable, une mise en balance d'incommensurables. Et donc la décision qui préside à ces choix est de l'ordre de la décision pure. C'est une décision politique, même si elle n'est pas prise par les responsables politiques, et même si elle n'est pas considérée comme telle par ceux qui la prennent.

En fait, il s'agit de désigner un équilibre. Un équilibre pour lequel il n'y a pas de référence fixe. Tout est question de limites, de limites acceptées, de limites acceptables, qui correspondent aux valeurs de la société au moment de la décision, à une définition de ce que serait donc, à un moment donné, le bien.

Ces valeurs, ces définitions sont, bien sûr, très évolutives.

De tels choix, sans filet, puisque sans références objectives qui puissent les cautionner, sont très difficiles à assumer dans le contexte socio-culturel qui est le nôtre. En effet, ces choix qui ne peuvent se référer à une quelconque objectivité, sont difficilement justifiables, peuvent sans cesse être remis en cause, et sont en conséquence très fragiles. J'avancerai ici volontiers l'hypothèse que le rôle conféré à la science et à la technique en matière de sécurité relève en fait de cette nécessité de trouver une référence, une légitimité rationnelle et donc indiscutable à ces choix qui sont sociaux. Dans ce cas-là, s'en remettre à la rationalité scientifique, c'est un peu faire comme si ces choix n'existaient pas, ou du moins, comme s'ils s'imposaient d'eux-mêmes, comme si c'était l'état du savoir technique qui fixait en quelque sorte les limites de la protection.

On pourrait presque dire ici que le pouvoir tente de s'indexer au savoir.

Cette transformation de ce qui est de l'ordre du politique en ce qui apparaît comme une décision simplement technique s'opère par de multiples voies, par un jeu complexe d'équivoques ou d'ambiguités entre les divers acteurs sociaux, techniciens et politiques, impliqués dans l'adoption de mesures de sécurité.

On peut citer un exemple de ces modes de passage du politique au technique: l'application de la norme de protection décennale en matière d'assainissement pluvial qui, bien que non imposée dans les textes, fonctionne en fait comme une règle quasi-coutumière. Cette norme est le produit d'un équilibre complexe entre de multiples enjeux : disponibilités financières des communes, niveau d'aspiration des citoyens à la sécurité, intérêts économiques multiples. Elle condense, elle synthétise un ensemble de choix sociaux implicites en quelque sorte et dont personne ne pourrait définir les termes de manière explicite.

Pourtant, dans la pratique, l'application de cette norme est bien souvent considérée comme une pure règle technique, comme une règle de l'art que l'ingénieur possèderait dans son bagage de compétence, et que le politique lui laisserait appliquer.

L'application de cette norme comme une règle technique dispense d'envisager l'éventualité que des risques ne sont pas couverts par son application. Elle invisibilise le fait que le choix de la protection est aussi un choix de nonprotection.

Pourtant, lorsqu'un événement se produit, d'une 
ampleur supérieure à celle pour laquelle les ouvrages ont été dimensionnés, les acteurs sociaux sont clairement confrontés au fait que les dommages subis sont la conséquence des décisions de protection et donc de nonprotection, qu'ils ont prises antérieurement et qui engagent leur responsabilité.

J'ajouterai à cela que le fait que de nombreux responsables politiques interprètent la protection contre un risque de fréquence décennale comme la certitude d'une protection absolue pendant 10 ans (et non pas comme la protection, vis-à-vis d'un événement ayant une chance sur dix de survenir dans l'année), ne me semble pas entièrement imputable à un manque de connaissance ou à la difficulté que rencontre un profane pour saisir des concepts mathématiques.

Penser ainsi permet aussi, me semble-t-il, de minorer l'enjeu de la décision. Celle-ci apparaît alors, non point comme concourant à diminuer la probabilité d'occurrence des événements dommageables mais comme un choix de protection absolue, pendant quelques années, choix qui n'engage à rien puisqu'il est provisoire et qu'il pourra être corrigé dans les années à venir.

C'est un tel jeu entre acteurs politiques et techniciens que fait brutalement éclater l'avènement d'une catastrophe comme celle du 3 octobre dernier à Nîmes. Par son ampleur, cette catastrophe met en évidence que des risques de cette nature ne sont pas couverts, et que cette nonprotection résulte d'un choix qui, s'il n'a pas été opéré en tant que tel, est en tout cas implicite.

Il semble que les réactions paradoxales que l'on a pu constater de la part des responsables politiques et de la population face à cet événement révèlent l'énorme difficulté à accepter cet état de fait.

A titre d'exemple, on peut citer une déclaration du maire de Nîmes, qui affirme, tout de suite après la catastrophe, que cet événement est tout à fait exceptionnel, qu'il était totalement imprévisible et qui assure dans la même phase que tout sera mis en œuvre pour qu'un tel événement ne se produise jamais plus.

Coexistent ainsi dans une même déclaration :

- la reconnaissance que la sécurité absolue est impossible ;

- et, l'affirmation que néanmoins, dans l'avenir, une telle sécurité pourra être garantie.

Sont ici juxtaposées deux affirmations contradictoires. Mais le fait que l'une réfère au passé et que l'autre s'applique à l'avenir permet de dissocier au moins provisoirement les deux termes du paradoxe.

On a pu enregistrer aussi de telles réactions paradoxales de la part de la population, des habitants. Au cours des interviews que nous avons pu réaliser quelques jours après la catastrophe, nous avons constaté que les réactions se partageaient, et quelquefois chez les mêmes individus, entre plusieurs sentiments contrastés :

- premièrement le sentiment diffus que l'événement était de ceux vis-à-vis desquels il est très difficile de se prémunir, ou, du moins, dont il faut rechercher l'origine dans une responsabilité commune et partagée; ce sentiment s'est exprimé en particulier par la relative cohésion, et par la relative solidarité de la population avec les pouvoirs publics dans les premiers jours qui ont suivi la catastrophe. Il explique peut-être aussi le caractère assez feutré des débats et des polémiques sur les causes de la catastrophe, du moins dans un premier temps.

- en deuxième lieu, un sentiment tout à fait opposé, la recherche de responsabilités tous azimuts, d'un coupable, d'une faute, d'une erreur. Sont alors souvent incriminés les responsables politiques à qui l'on reproche d'avoir repoussé les bonnes solutions techniques, garantes du bien commun, au profit d'intérêts particuliers. La rumeur des 100 morts qu'on aurait cachés à la population a, dans ce contexte, une fonction, celle de conforter l'existence d'une faute que l'on tenterait à tout prix de masquer.

C'est à mon avis de telles conduites paradoxales que l'on risque d'observer dans les semaines à venir lorsqu'il s'agira de tirer les enseignements de l'expérience pour définir une politique de sécurité. Et peut-être faudra-t-il s'attendre à une dramatisation de ce jeu d'équivoques feutrées entre techniciens et politiques ou entre responsables politiques et population. La fiction d'une protection indolore et absolue s'effondre, en effet, sous le choc d'une telle catastrophe, en même temps que sont mises à jour les limites du seul recours à la science et à la technique pour garantir la sécurité.

La science ne peut plus se substituer au politique pour réaliser des choix. Loin d'invisibiliser les arbitrages à effectuer, elle tend, au contraire à les révéler et à les renvoyer à la responsabilité politique.

En outre, elle admet et fait savoir que subsistent de grandes marges d'incertitude quant à la prévision d'un tel événement, compte tenu de sa rareté.

On peut se demander comment, dans ces circonstances, vont pouvoir réagir les instances politiques, orphelines de la protection que leur apportent habituellement les techniciens, et sommées, donc, de prendre seules une double responsabilité :

- celle de reconnaître l'impossibilité de garantir une protection totale qui obligerait à rayer une partie de Nîmes de la carte ; celle d'accepter, en conséquence, de faire courir aux Nîmois un certain nombre de risques, et de renoncer ainsi à être le garant de la sécurité.

- celle d'assumer le coût social, politique et économique d'une protection qui restera incomplète, mais qui conduirait, peut-être, à remettre largement en cause le type d'urbanisation qui a prévalu jusqu'à présent. Cela, dans un contexte très fortement marqué par l'incertitude, et sans pouvoir donc être totalement sûr de l'efficacité des solutions retenues.

La question reste largement ouverte; il ne s'agit pas de faire ici de la prospective politique, mais on peut imaginer que la manière dont se dénouera une telle situation se situe entre deux issues extrêmes :

- soit le renforcement du processus d'invisibilisation du caractère politique des décisions tel que je l'ai évoqué précédemment ;

- soit au contraire, à l'autre extrême, une crise de légitimité de la puissance publique, confrontée à l'impossibilité d'assumer, face aux risques, sa fonction providentielle. 


\section{Discussions}

\section{F. FRUCHART, Compagnie nationale du Rhône:}

En matière de probabilité de crue, le risque d'une chance sur dix par an de voir le réseau insuffisant semble acceptable. Cependant, c'est une présentation trompeuse. Quelle réponse aurait-on, si l’on demandait aux élus le risque qu'ils veulent bien prendre pour les dix prochaines années par exemple? Si l'on fait le calcul, risque d'une crue décennale en dix ans $\left(1-0,9^{10}\right)$, cela fait environ deux chances sur trois. Seraient-ils d'accord pour prendre ce risque?

Pour les grands barrages, c'est un peu la même chose. Ils sont protégés contre la crue millennale, le risque semble minime. Cependant, le risque pris pendant une durée de vie de cent ans est de 1 sur 10, ce qui est loin d'être négligeable étant donné le risque encouru.

J.-C. HÉmaln, Laboratoire central des Pontset-Chaussées

Je dirai même : on peut, peut être, aller au-delà.

C'est vrai qu'on a un problème de communicabilité lorsqu'on parle de période de retour parce que le concept mathématique de période de retour n'est pas en soi évident à percevoir. Il suffit d'en prendre pour preuve ce qui a été dit au sujet de Nîmes. Même si les chiffres bruts de période de retour étaient plus ou moins bons ou mauvais, le plus important, c'est ce qu'on a entendu dire : si la période de retour est 500 ans, on est tranquille pour 500 ans.

Non seulement, il y a ces confusions-là, mais si on veut avancer, je crois qu'il faut aller au-delà. Il faut remettre en cause cette période de retour et parler de risques. Les chiffres que je vous ai présentés ce matin sont surtout relatifs à la pluie. Mais il est bien évident que ce qui intéresse la population ce sont les conséquences de cette pluie, et lorsque la période de retour se situe bien au-delà de 10 ans, ces conséquences se traduisent par des dégâts. Les surfaces concernées sont très importantes et la notion de période n'a plus de sens en soi.

$\mathrm{Ne}$ parlons plus de cette sacro-sainte période de retour sinon pour des objets ponctuels, mais parlons de risque, parlons d'aménagement de l'espace pour gérer, pour assumer ces risques, parlons de niveau de protection, du risque qu'on veut bien courir et non pas de celui contre lequel on se protège.

A. BACHOC, CERGRENE, Ecole nationale des Pontset-Chaussées

Je pense aussi qu'il est important d'aborder la question en termes de risques extrêmes, ce qui est rarement fait dès qu'on parle de probabilité décennale, etc... Il est important aussi de faire des projections pour le cas des événements tout à fait exceptionnels. Pour voir dans quelle mesure on peut minimiser leur impact, sans bien sûr pouvoir effacer complètement celui-ci.

Et Nîmes peut offrir un support à la communication vis-à-vis des élus dans ce domaine-là.

\section{J.-C. HÉMAIN :}

Sans vouloir monopoliser la parole, une petite précision à ce que vient de dire André. Une lecture attentive de la circulaire montre qu'il est écrit en toutes lettres dans le paragraphe "Introduction aux conceptions générales" qu'il est tout à fait souhaitable de s'interroger sur le devenir des eaux pluviales lorsqu'on atteint des niveaux de probabilités bien au-delà de celui qui est retenu pour le projet.

Ce n'est pas mis en exergue, ce n'est pas ce qui est retenu, ce n'est pas le tableau que le technicien a sur son bureau pour faire ses calculs, il n'en reste pas moins que c'est écrit.

\section{DUMAY, BCEOM}

En matière de stratégie d'aménagement, il me semble utile de distinguer :

- les réseaux secondaires qui servent à l'assainissement et à évacuer les eaux pluviales (ordinaires); ils ont une incidence éventuellement assez réduite vis-à-vis de dommages aussi bien sur les biens que sur les personnes.

- les réseaux primaires qui servent à évacuer les crues d'un volume déjà important, pour lesquels on doit avoir une notion de période de retour tout à fait différente.

Je voudrais ajouter à cela qu'il me semble qu'on voit actuellement une inflation galopante à l'issue de ce qui s'est passé à Nîmes et qu'on arrive maintenant à proposer, peut-être, des surdimensionnements de réseaux. Il me semble qu'il ne faut pas, là, aller au-delà d'une certaine limite, mais qu'on pourrait par contre utiliser des infrastructures de surface liées à l'aménagement urbain, les infrastructures routières notamment, ce que nous avons appelé au BCEOM les transparences hydrauliques qui permettent d'écouler les eaux en dehors des réseaux.

En matière d'inflation galopante sur l'estimation des débits, je reviendrai un petit peu sur ce qu'a dit ce matin Jean-Claude HÉmain au sujet de l'estimation de la période de retour sur Nîmes. L'étude du LHM de Montpellier a mis en évidence que ponctuellement on peut avoir des hauteurs pluviométriques importantes qui correspondent à des périodes de retour élevées. Il faut savoir si cette pluviométrie importante sur des zones de superficie assez faible peut se produire sur des surfaces relativement grandes, comme cela s'est produit à Nîmes. Or, sur Nîmes, il y a l'intensité pluviométrique et l'analyse qu'on en a faite sur la base des courbes intensité-durée-fréquence, il y a donc aussi les paramètres "durée» et "fréquence», mais il faudrait y ajouter un troisième paramètre très important, la dispersion spatiale. A Nîmes, on a eu un phénomène exceptionnel et qui n'était pas ponctuel, qui intéressait une région entière. La gravité de l'événement est due au fait que le volume engendré était très important.

Or des pluies de période de retour très élevées, de niveau millénale, on en a régulièrement, je dirai même annuelle- 
ment, mais elles affectent des zones de 1 ou 2 hectares. Elles ont donc un effet à l'aval relativement limité puisque ces bassins versants sont réduits.

Dans la mesure où la surface de la zone touchée est beaucoup plus importante, il serait nécessaire de prendre cela en compte dans l'estimation de la période de retour. Et pour moi, l'événement survenu à Nîmes a une période de retour nettement supérieure à 100 ans.

Bien sûr, comme le disait Jean-Claude Hémain, cette estimation est difficile à obtenir, mais je crois qu'on peut avoir des estimations nettement divergentes avec des approches scientifiques faites dans un cadre différent.

La question est posée là-dessus, mais il me semble que l'estimation des périodes de retour des débits sur Nîmes est sous-estimée.

\section{E. JACOBSEN, Cabinet Sauveterre.}

Ma réflexion va dans le sens de celle qui vient d'être faite. Je pense aussi qu'il ne faut pas généraliser les conceptions hydrauliques à partir de phénomènes exceptionnels. La plupart des problèmes d'assainissement à résoudre ne présentent pas cette accumulation de difficultés (pentes très fortes, grand bassin versant en entonnoir abrupt) rencontrées ici...

\section{RENARD, Direction régionale de l'Equipement d'Ile de} France :

Je voudrais me faire l'écho d'une discussion que nous avons eue à notre table à midi :

Les techniciens, au sens noble du terme, ne peuvent-ils pas apporter des réponses résultant de deux démarches parallèles :

- l'une qui est classique: en tant que maîtres-d'œuvre, nous devons dimensionner des ouvrages, donc choisir une période de retour, et économiquement elle ne peut guère excéder 10 ans ;

- une autre, à mener parallèlement, consistant à analyser comment va réagir ce réseau, dimensionné pour 10 ans, face à un événement exceptionnel, comme celui de Nîmes : où va passer l'eau? dans quelle zone risque-t-elle d'être bloquée?

Il convient alors d'imaginer des règles d'urbanisme ou d'aménagement, en évitant par exemple que certaines rues soient trop étroites pour permettre l'écoulement de surface au moment où la ville sera soumise à un événement exceptionnel.

On peut faire à cet égard un parallèle avec les règles d'aménagement fluvial dans les zones inondables. Dans les zones de grand écoulement en période de crue, on interdit, par exemple, de mettre des barrières, des clôtures, ...

J'ai été surpris de voir sur des images de Nîmes, dans certaines rues, des enchevêtrements de voitures les unes sur les autres. Il y a peut-être des règles à préconiser pour éviter des vitesses trop élevées ponctuellement, susceptibles d'entraîner des véhicules, du mobilier urbain, etc., qui en s'agglutinant bloquent des rues étroites se comportant alors comme des entonnoirs bouchés.

Je proposerai donc qu'on mène en parallèle des études classiques de dimensionnement et des études de réaction de ce projet face à des événements exceptionnels afin d'apprendre à minimiser leur impact.

J.-M. SoтtY, Service technique de la ville de Marseille

Pour répondre au problème de la fréquence de protection, je voudrais poser la question de façon globale en essayant d'ouvrir le débat.

Il me semble que le technicien, ou le gestionnaire des eaux pluviales au sens large, dispose a priori de trois outils :

— un outil technique, par le biais des aménagements;

- un outil réglementaire, par le biais des réglementations

du POS, du règlement d'assainissement, etc... qui est surtout préventif ;

- et un outil correctif, qui consiste, à partir du moment où il y a eu des inondations, à mettre en place une structure de secours permettant d'organiser au mieux l'ensemble des actions.

Et il me semble qu'il n'y a pas non plus une seule fréquence de protection à définir, mais trois. Si j'essaie de caricaturer mes propos, d'être un peu entier par rapport à ce que je pense, je dirai qu'il y a :

- un domaine d'événements courants, dans lesquels, raisonnablement, un technicien peut avoir la prétention d'assurer une bonne protection : c'est probablement pour des périodes de retour de 10 ans, 20 ans, 50 ans, suivant la place et l'argent dont on dispose. C'est la situation initiale : - un deuxième domaine se situant entre 10 ans et 100 ou 200 ans, qui est le domaine du réglementaire, sur lequel le technicien prévient qu'il existe un lit majeur inondable et il essaye d'œuvrer à une meilleure adéquation entre l'urbanisme et l'écoulement exceptionnel ;

- enfin un troisième domaine où, quoiqu'on fasse, l'événement est grave, et il faut réfléchir à l'organisation des secours sur tous les plans.

Il me semble qu'il est préférable, plutôt que de se focaliser sur la fréquence de protection que peut assurer tel aménagement, d'étudier aussi les autres fréquences, pouvant varier de zéro à l'infini, en les regroupant en trois domaines.

Et chaque fois, il y aura une étude de cas à traiter pour déterminer où on placera les frontières entre ces trois domaines.

J. GRUAT, Institut de mécanique des fluides de Toulouse Pardonnez à un professeur d'intervenir dans une discussion aussi technique, mais je voudrais reprendre l'idée de Monsieur RENARD. C'est ce qui se fait pour les grands ouvrages de protection, ou pour les grands barrages.

Si vous le permettez, je citerai deux anecdotes, très rapidement: une fois, j'ai travaillé pour la protection contre les crues de la ville de Périgueux. Les débits pour lesquels on a travaillé sont de $800 \mathrm{~m}^{3} / \mathrm{s}$. Si la prochaine crue a un débit de $795 \mathrm{~m}^{3} / \mathrm{s}$, tout le monde dira : "les hydrauliciens sont des gens de génie ", mais si elle fait $805 \mathrm{~m}^{3} / \mathrm{s}$ tout le monde dira : " les hydrauliciens sont des idiots, ils auraient relevé les digues de $10 \mathrm{~cm}$, nous aurions été à l'abri ".

J'ajoute une dernière remarque, c'est que dans les grands ouvrages on essaie de tout imaginer, non seulement les 
périodes de retour des crues, mais également les périodes de retour des pannes. Si on veut contrôler l'évacuation des crues par des vannes, ces vannes peuvent rester coïncées. J'ai travaillé une fois sur un projet où il était question d'abord d'évacuer $2500 \mathrm{~m}^{3} / \mathrm{s}$, puis ensuite on est passé à $2700 \mathrm{~m}^{3} / \mathrm{s}$, tout en supposant que cette crue arriverait quand le barrage était plein. Puis ensuite on a dit qu'une vanne, sur les trois, peut rester coïncée. Puis on en est resté là, parce qu'il restait à imaginer ce qui se passerait si les trois vannes restaient coïncées.
Donc, de toute façon, on sait qu'il y a un seuil arbitraire à partir duquel il faut bien qu'on s'arrête.

Mais je crois que l'idée des interventions de Monsieur RENARD et de Monsieur SotTy qui consistent à fixer des paliers de protection qui ne sont pas traités de la même façon me semble être une bonne façon de procéder.

\section{F. CALVET}

Le débat est donc ouvert, mais je suis au regret de devoir le clôre, le risque étant de ne pouvoir terminer ce soir.

Photo Fahra - Paris-Match

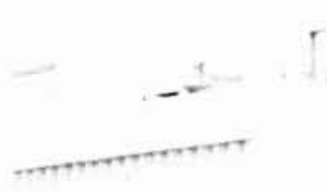

II

$1=1$
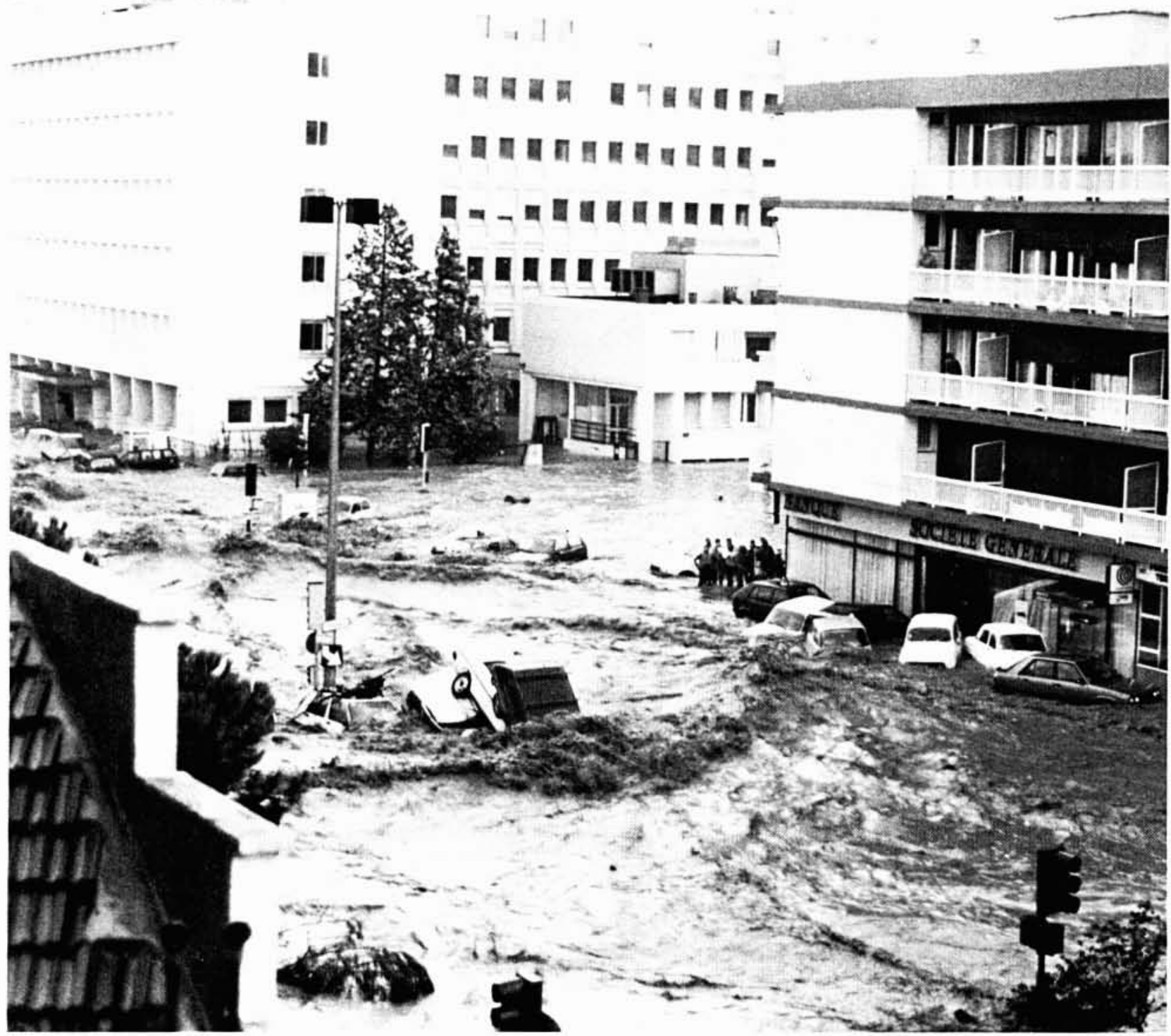

LA HOUILLE BLANCHE/ $\mathrm{N}^{\circ}$ 6-1989 


\section{La section Hydrologie urbaine de la Société hydrotechnique de France}

L'intérêt que porte la Société hydrotechnique de France (SHF) au domaine de l'hydrologie urbaine est manifeste et déjà ancien, comme en témoignent diverses manifestations des deux décennies passées ; et c'est pour répondre à cette attente, formulée notamment au lendemain des $\mathrm{XVII}^{\mathrm{e}}$ Journées de l'hydraulique à Nantes, en 1982, que fut décidée la création d'une section Hydrologie urbaine au sein de la division Aménagement des eaux de la SHF.

L'enfantement fut difficile et le bébé mit quelque temps pour trouver son équilibre; mais aujourd'hui plein de vigueur, il marche, mieux il progresse, et on doit lui souhaiter une adolescence pleine de découvertes et d'enseignements.

A la création de la section Hydrologie urbaine, ses animateurs ont fait un pari ambitieux : "créer un vaste réseau de correspondants afin de promouvoir une politique d'échange d'informations et d'expériences, de recensement des besoins émanant des hommes de terrain, de promotion de la recherche et des techniques innovantes, ... en résumé, favoriser le double flux de transfert des compétences et des expériences entre chercheurs, projeteurs et gestionnaires dans le domaine de l'eau et de l'aménagement urbain ".

Cette démarche répond à plusieurs objectifs pressentis par les animateurs et amendés par les premières propositions des correspondants.

La satisfaction d'objectifs aussi ambitieux exige l'engagement de chacun et du plus grand nombre, à commencer par les animateurs (voir encadré).

La démarche "réseau de correspondants » bénéficie de deux supports pour se concrétiser :

- la publication périodique ( 2 à 3 fois par an) d'un bulletin que la section Hydrologie urbaine diffuse gratuitement auprès des membres du réseau de correspondants. Au sommaire on peut trouver des rubriques variées : articles de fond, chronique, vient de paraître, nouvelles brèves, humeurs, qu'en est-il Auguste ?... Ce bulletin c'est un peu l'auberge espagnole, il a pour ambition de faciliter les échanges, c'est-à-dire qu'il n'aura d'intérêt que par ce que vous lui apporterez. Nous sommes très demandeurs d'articles, d'informations brèves sur des manifestations passées ou à venir, de tous éléments d'information qui vous sembleraient intéressant de faire partager à la communauté scientifique et technique ;

- la tenue de journées techniques d'échanges, chaque année, sur des sujets librement choisis par le réseau de correspondants ; c'est-à-dire, que nous ferons appel à vous au travers de notre bulletin pour cerner vos centres d'intérêt. Cette journée se concrétise par l'édition a posteriori, dans les meilleurs délais, de l'intégralité des communications et des débats. Deux journées ont déjà eu lieu :

- en 1987, le 8 décembre: Thérapeutiques nouvelles en assainissement pluvial ;

— en 1988, le 30 novembre: Ouvrages de contrôle hydraulique en réseaux d'assainissement.

Au cours de cette dernière journée, au lendemain des pluies catastrophiques qui se sont abattues sur Nîmes, il nous a semblé utile d'aborder ce sujet d'actualité. Au-delà de l'amplification médiatique de cet événement, la catastrophe de Nìmes a permis d'enclancher un certain nombre de réflexions ou de réactions dans tous les milieux " parties prenantes " de l'aménagement urbain : technique, politique, sociologique, économique, urbanistique... Les exposés précédents sont extraits du programme présenté le 30 novembre 1988 lors de notre journée d'échanges.

Pour tous renseignements complémentaires sur les activités de la section Hydrologie urbaine, contactez :

André BACHOC-CERGRENE-I.M.F. - Groupe

Ecoulement diphasiques, 31400 Toulouse. Tél. : 61285800.

Francis Calvet - SCP/ID - Société du Canal de Provence - Le Tholonet,

13603 Aix-en-Provence Cedex 1. Tél. : 42239850 .

Claire Cogez - Conseil Général de Seine-St-Denis, Hôtel du Département,

1, avenue Youri-Gargarine,

93000 Bobigny. Tél. : 48956060 .

Jean-Claude HÉmAIN - L.C.P.C. - Centre de Nantes

- Division Eau,

B.P. 19 ,

44340 Bouguenais. Tél. : 40845800 .

Daniel RENARD - D.R.E.I.F./D.E.R.U. - Direction régionale de l'équipement d'Ile de France,

21, rue Miollis,

75732 Paris Cedex 15. Tél. : 40618359.

Claude ThiBAud - Service de l'Assainissement, Hôtel-de-Ville,

72039 Le Mans Cedex. Tél. : 43474685. 